Reprod. Nutr. Dévelop., 1987, 27 (1 B), 295-296.

\title{
Effet d'un excès alimentaire de leucine sur la cinétique postprandiale de l'insuline plasmatique chez l'agneau préruminant
}

\author{
Isabelle PAPET, D. BREUILLÉ, D. ATTAIX, J. GRIZARD, M. ARNAL \\ Laboratoire d'Etude du Métabolisme Azoté, \\ I.N.R.A. et C.N.R.S. U.A. 041123 , Theix 63122 Ceyrat, France
}

Summary. Postprandial plasma insulin was measured in lambs fed a milk substitute in which L-leucine content was 2.3 (control) and then increased to 5.2 or $10.6 \mathrm{~g} / 100 \mathrm{~g} \mathrm{D} \mathrm{M}$. A significant enhancement of postprandial plasma insulin, compared to controls, was observed only when the diet contained $10.6 \mathrm{~g} \mathrm{~L}$-leucine $/ 100 \mathrm{~g} \mathrm{DM}$. This suggested that the effects of a dietary supplement of L-leucine on protein metabolism may be mediated by insulin only when this excess is high.

La L-leucine stimule la sécrétion d'insuline (hormone régulatrice du métabolisme protéique) in vivo chez le rat (Mazzaferri et al., 1983) et l'homme (Eriksson et al., 1983). De plus la L-leucine seule a, au moins in vitro, un rôle régulateur du métabolisme protéique (Walser, 1984). L'étude du métabolisme des acides aminés à chaîne ramifiée et plus particulièrement de la L-leucine, chez l'agneau préruminant, nous a conduit à préciser l'effet d'excès alimentaires de leucine sur les cinétiques postprandiales de l'insuline et du glucose plasmatiques chez cette espèce.

Matériel et méthodes. Dix agneaux mâles de race Limousine reçoivent ad libitum un lait de remplacement dont la matière sèche (MS $=16 \%$ ) contient $24 \%$ de protéines $(N \times 6,25)$ apportées exclusivement par de la poudre de lait écrémé et $2,3 \%$ de leucine. Les consommations et croissances moyennes sont respectivement de $270 \mathrm{~g} \mathrm{MS} / \mathrm{j}$ et $280 \mathrm{~g} / \mathrm{j}$ entre les âges de 1 et 2-3 semaines. A l'âge de 2-3 semaines, les agneaux sont séparés en 2 lots $A$ et $B$ et reçoivent, pendant 2 jours $(J+1$ et $J+2)$, le lait supplémenté en L-leucine contenant alors respectivement 5,2 et $10,6 \mathrm{~g}$ de L-leucine/100 g MS (tabl. 1). Pendant les 4 jours expérimentaux $\mathrm{J} 0, \mathrm{~J}+1 ; \mathrm{J}+2$ et $\mathrm{J}+\mathrm{n}$ des prélèvements de sang (veine jugulaire) sont effectués avant et après un repas de 300 à $500 \mathrm{~g}$ de lait, durant de 0,5 à $1 \mathrm{~h}$ et suivi d'une période de 3,5 à $4 \mathrm{~h}$ sans aliment. L'insuline plasmatique est dosée par radioimmunologie selon la méthode du double anticorps à l'aide d'insuline humaine comme standard. Le glucose plasmatique est déterminé par la méthode à la glucose déshydrogénase.

TABL. 1. - Schéma expérimental.

\begin{tabular}{|c|c|c|c|c|c|}
\hline \multirow{2}{*}{ Lot } & \multirow{2}{*}{$\begin{array}{c}\text { Nombre } \\
\text { d'agneaux }\end{array}$} & \multicolumn{4}{|c|}{ Teneur du lait en leucine $(\mathrm{g} / 100 \mathrm{~g} \mathrm{MS})$} \\
\hline & & J 0 & $J+1$ & $J+2$ & $J+n$ \\
\hline A & 3 & \multirow{2}{*}{2,3} & 5,2 & 5,2 & \multirow{2}{*}{2,3} \\
\hline B & 7 & & 10,6 & 10,6 & \\
\hline
\end{tabular}

$\mathrm{J} 0, \mathrm{~J}+1, \mathrm{~J}+2: 3$ jours consécutifs; $\mathrm{J}+\mathrm{n}: 2$ à 5 jours après $\mathrm{J}+2 ; \mathrm{J} 0, \mathrm{~J}+\mathrm{n}$ : jours témoins.

Résultats et discussion. Comme chez le veau préruminant (Grizard et al., 1982), l'ingestion du régime témoin provoque une augmentation importante de la teneur plasmatique en insuline entre 0,5 et $2 \mathrm{~h}$ après le début du repas (fig. 1a). 
La teneur plasmatique en glucose évolue dans le même sens que celle d'insuline (fig. 1b). Il existe une corrélation élevée entre les teneurs plasmatiques en insuline et en glucose observées avec le régime témoin $\left(r^{2}=0,63\right)$. L'évolution postprandiale de la teneur plasmatique en insuline n'est pas significativement modifiée lorsque le lait contient $5,2 \mathrm{~g}$ de L-leucine/100 g MS. En revanche l'ingestion du régime à $10,6 \mathrm{~g}$ de L-leucine/100 $\mathrm{g}$ MS s'accompagne d'une amplification de l'augmentation postprandiale de la teneur plasmatique en insuline. Cet effet est mis en évidence par le test de Wilcoxon (comparaison de données naturellement appariées) pour les temps 1 et $4,5 \mathrm{~h}(\mathrm{P}<0,05)$ et $2 \mathrm{~h}(\mathrm{P}<0,1)$ après le repas du jour $J+1$ et $1 \mathrm{~h}(P<0,1)$ après celui de $J+2$. Les surcharges de L-leucine ne modifient pas significativement la cinétique postprandiale de la teneur plasmatique en glucose. La L-leucine $(10,6 \mathrm{~g} / 100 \mathrm{~g} \mathrm{MS})$ serait directement responsable de l'accroissement de la teneur plasmatique en insuline postprandiale par rapport au régime témoin. Cette action stimulante a lieu malgré l'effet opposé de la réduction de l'ingestion de ce régime par rapport au régime témoin (Papet et al., 1987).

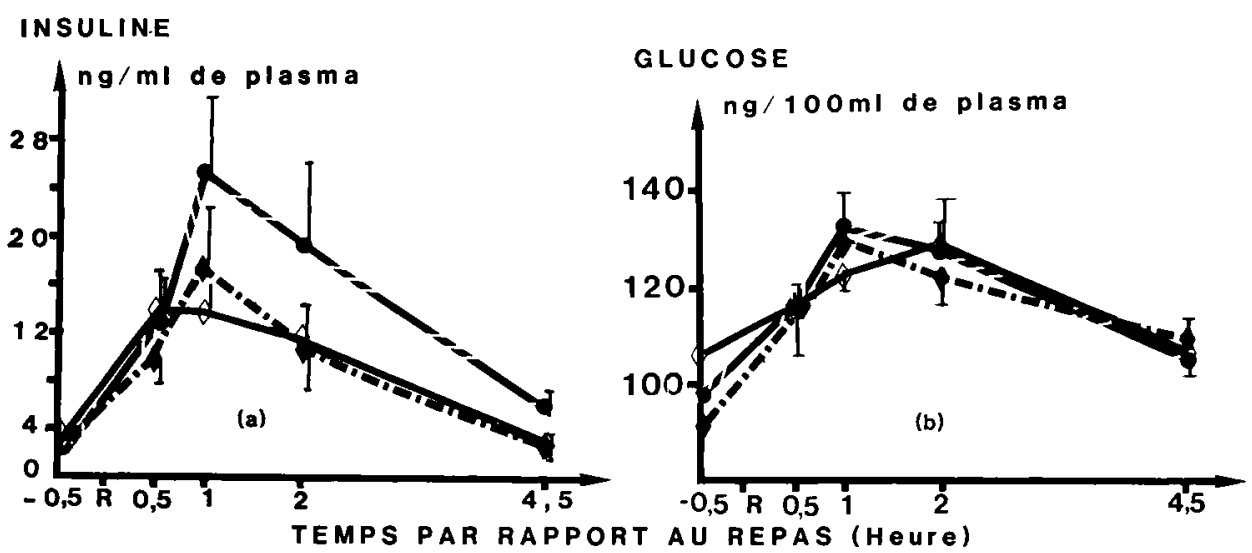

FIG. 1. - Evolution postprandiale des teneurs plasmatiques en insuline (a) et glurose (b) chez l'agneau préruminant recevant un lait de remplacement contenant $2,3(-\infty) ; 5,2(--)_{1}$ ou 10,6 $(---1) \mathrm{g}$ de leu/ $100 \mathrm{~g} \mathrm{MS}$.

Moyennes \pm écart-types ( $\mathrm{n}=3$ à 10$)$; ces moyennes sont calculées pour $\mathrm{J} 0, \mathrm{~J}+\mathrm{n}(-\bullet-\bullet)$ et pour $J+1, J+2(---)$ ou $(----)$.

L'effet insulino-secréteur de la L-leucine semble moins important chez l'agneau que chez le rat et l'homme. L'intervention des effets métaboliques de I'insuline dans l'explication des modifications du métabolisme des acides aminés à chaîne ramifiée ne semble devoir être prise en compte que pour des surcharges alimentaires de L-leucine très importantes.

Remerciements. - à J. P. Chacornac pour les dosages du glucose.

Eriksson L. S., Hagenfeldt L., Felig P., Wahren J., 1983. Clin. Sci., 65, 491-498.

Grizard J., Toullec R., Guilloteau P., Patureau-Mirand P., 1982. Reprod. Nutr. Dévelop., 22, $475-484$.

Mazzaferri E. L., Ciofalo L., Waters L. A., Starich G. H., Groshong J. C., Depalma L., $1983 . \quad A m$. J. Physiol., 245, E114-E120.

Papet 1., Breuillé D., Arnal M., 1987. Reprod. Nutr. Dévelop., 27, 293-294.

Walser M., 1984. Clin. Sci., 66, 1-15. 